\title{
CREENCIAS SOBRE JUEGO DE LOS PROFESORES EN EDUCACIÓN INFANTIL ${ }^{1}$ BELIEFS ABOUT GAME OF TEACHERS IN CHILDHOOD EDUCATION
}

\section{FASE 1}

\author{
Sandra Marcela Durán Chiappe ${ }^{2}$
}

Resumen

Este artículo presenta, de manera sucinta, algunas de las construcciones teóricas sobre los dos campos de conocimiento que estructuran la apuesta investigativa Creencias sobre juego de los profesores en Educación Infantil ${ }^{3}$, a saber: las creencias del profesorado y el juego.

Para abordar dichos campos de conocimiento, se diseñaron algunas preguntas que guían la investigación y el itinerario que conlleva al encuentro con autores que han desarrollado estudios y trabajos en torno a los campos mencionados y con quienes se entra en un diálogo permanente desde las apuestas que se hacen en la docencia y la investigación.

Palabras clave: creencias, juego, educación inicial.

Abstract

This article presents, in a succinct way, some of the theoretical constructions on both fields of knowledge that structure the investigative proposal Creencias sobre juego de los profesores en Educación Infantil, namely: the beliefs of the professorship and the game.

To approach the above mentioned fields of knowledge, there were designed some questions that guide the investigation and the itinerary that it carries to the meeting with authors who have developed studies and works concerning the mentioned fields and with whom start a permanent dialog from the proposal that are done in the teaching and the investigation.

Key words: beliefs, game, initial education.

Fecha de recepción: 10 de Octubre de 2010

Fecha de aprobación: 2 de Noviembre de 2010

${ }^{1}$ Proyecto de investigación elaborado en el marco de la tesis doctoral de la profesora Sandra Marcela Durán. Doctorado en Pedagogía Social. Universidad de Granada, España.

${ }^{2}$ Docente Investigadora Universidad Pedagógica Nacional. Magister en Educación. Candidata a Doctor en Pedagogía Social. Universidad de Granada, España.

${ }^{3}$ Equipo de investigación. Coinvestigadoras: Consuelo Martín Cardinal, Jenny Pulido. Monitoras: Luisa Castillo Restrepo, Mónica Gil Cardona. 
Para el grupo investigador existe un profundo interés en lo relativo a las acciones educativas que se desarrollan con niños y niñas en educación inicial, pues, en distintos escenarios del contexto colombiano, este ha estado caracterizado por el asistencialismo, al margen de las propuestas que dinamizan nuevas lógicas y maneras de asumir el trabajo con la niñez menor de dos años.

Es en este contexto en el cual cobra vigencia interrogar por las creencias de los docentes que trabajan en educación inicial, pues estudiarlas implica no sólo un acercamiento a su discurso, sino a su práctica.

En este sentido, algunas de las preguntas que orientan la apuesta investigativa son: ¿cuáles son las creencias sobre juego y la acción misma de jugar, que tienen los maestros que trabajan en el aula con niños en Educación Inicial?, dentro de las propuestas de juego del profesorado, ¿cuál es su rol, qué papel cumple el ambiente y los objetos, y qué manifestaciones se pueden identificar en los niños?

Con el objeto de construir un horizonte de sentido que permita abordar dichos interrogantes, se diseñan dos criterios para el reconocimiento de los trabajos investigativos:

- El período de tiempo en el que se concentra la búsqueda, comprendido entre 1990 y 2009.

- Las investigaciones se hubiesen desarrollado con maestros de Educación Inicial y niños, menores de 6 años.

\section{Las creencias en el pensamiento del profesorado}

Rokeach (1968), citado por Argoz (1997) hace uno de los principales aportes al concepto de creencia que se utiliza en educación, en el cual define creencia como:

Una proposición simple, consciente o inconsciente, inferida de lo que una persona dice o hace, capaz de ser precedida por la frase: -yo creo que[...] que describe el objeto como verdadero o falso; correcto o incorrecto; lo evalúa como bueno o malo y se predispone para actuar posiblemente de diferentes formas bajo diferentes condiciones.

Clark (1986) y Peterson (1990) muestran en su estudio sobre el tercer problema, que para referirse a las creencias se utilizan términos como: perspecti- va personal del docente, principios de práctica, teorías implícitas, conocimiento práctico.

De la misma manera, Gallego (1991) en su revisión sobre la investigación de los pensamientos del profesor, afirma que el término de creencias: "compite con otros términos (esquemas, perspectivas, concepciones) y no siempre es utilizado con el mismo significado".

Un ejemplo de esta polisemia y complejidad de este campo de investigación se puede encontrar en Spode y Sracho (1988), citado por Argóz (1997) quienes manifiestan: que "estas teorías implícitas de los profesores, denominadas creencias del profesor, constructos curriculares, influencian el modo en que los profesores perciben las situaciones de clase e interpretan el marco educativo".

Los orígenes del término creencias se remontan a Dewey (1933-1989), para quien las creencias son ideas adquiridas de otros, que se aceptan como una idea común sin que la persona examine el asunto:

Estos pensamientos (creencias) son prejuicios, es decir, ideas preconcebidas no conclusiones a las que se haya llegado como resultado de la actividad personal, como la observación, el recuerdo y el examen de la evidencia. Aunque son correctos, su corrección es puramente casual, al menos en lo que respecta a la persona que los sostiene.

Fandiño (2004), reflexiona lo planteado por Dewey, aclarando que este autor no niega el valor de las creencias sino el proceso por el cual se accede a estas, en consecuencia propone que sea la deliberación y no otra cosa, lo que soporte las creencias.

De acuerdo con lo anterior, se puede decir que la creencia en su inmensa complejidad no puede llegar a identificarse y comprenderse exclusivamente a través de la práctica del docente, o solamente con su discurso, es a través de la articulación entre estos dos procesos como se puede llegar hacer una aproximación al pensamiento del profesor, por tal motivo, para el estudio de las mismas son necesarios los enfoques interpretativos, que superan las metodologías de escalas, ya que brindan la posibilidad de conocer no solamente lo que el profesor dice sino lo que hace y los significados según el contexto. 
Ya el mismo Marcelo (1992), expresaba que el hecho de acceder a los significados que los profesores atribuyen a los "objetos" de su trabajo puede ayudar a explicitar la comprensión de las conductas ante determinada situación de enseñanza. Los significados se constituyen en pieza clave para comprender la manera como un sujeto ve el objeto, el modo como actúa con respecto al mismo y también la forma en la cual se dispone a hablar acerca de él. Esto quiere decir que el significado de juego construido por el profesor, determina sin lugar a dudas sus perspectivas de acción.

Igualmente Fenstermacher (1989), afirma que conocer las creencias de los profesores ayuda al maestro a comprender mejor su práctica y a resignificarla. Indagar entonces, sobre las creencias, implica un acercamiento a la práctica de los docentes y a su discurso, para encontrarle sentido a sus actuaciones. En esa medida Sola (1999), analiza a través del estudio de la reforma experimental de la EGB (Andalucía, España), las creencias y su importancia en el pensamiento del profesor, puesto que son estas las que orientan o inciden en su actuación profesional, según este autor la creencia se puede entender como un estado interno, una cierta "disposición" para actuar. La persona cree en algo y en tal sentido tiene la disposición para actuar coherentemente.

Uno de los aspectos más valiosos del trabajo que se cita anteriormente, y que se constituye como un aporte contundente para la presente investigación, es justamente el que explicita el asunto de la identificación y revisión de las creencias, mediante la reflexión y la deliberación, como un elemento clave para el desarrollo profesional y este precisamente es un asunto realmente importante en tanto no se trata exclusivamente de identificar y comprender las creencias de las maestras con quienes se desarrolla la investigación, sino además de ponerlas en diálogo con las creencias de las otras maestras y del mismo sujeto que investiga para desde allí generar un proceso reflexivo de las mismas. En ese sentido es pertinente reconocer que en la identificación de las creencias es primordial la contrastación con otros puntos de vista, otras opiniones, por lo tanto es decisivo el trabajo en grupos de profesores, de tal manera que se exterioricen los propios argumentos en un proceso de deliberación.
Del mismo modo, para llegar hacer un análisis riguroso sobre la manera como las creencias se originan y mantienen, es muy importante exponer y reconocer las razones de orden cultural, social, económico, político, que las personas tienen para creer y los motivos que las impulsan a ello, con el fin de poder explicar su permanencia. Al mismo tiempo, si se pretende llegar a una transformación de las creencias, es necesario un proceso de deliberación, a partir de la reflexión crítica y conjunta de manera que se pueda reconstruir el pensamiento y analizar las causas, las razones y los motivos que se tienen para mantenerlo.

En relación con lo anterior, Martínez (1994), en su estudio sobre Las teorías implícitas y la resolución de problemas de la enseñanza: el pensamiento del profesorado en formación inicial, hace énfasis por una parte, en el conocimiento de las características de las teorías implícitas del futuro profesorado sobre la enseñanza (como síntesis de conocimiento práctico) y por otra parte, al conocimiento de los mecanismos de inducción de dichas teorías en la toma de decisiones a partir de las tareas (problemas de enseñanza).

Gran parte de las inquietudes de este estudio, consisten precisamente en comprobar cómo se conforman las teorías implícitas del profesorado en formación, verificar si este tipo de conocimiento constituye la génesis de su toma de decisiones, indagar si el conocimiento práctico del profesorado sin experiencia en el aula, se conforma con sus experiencias escolares pasadas y con el conocimiento académico compartido durante su formación, en síntesis, explorar ese marco de referencia al que los sujetos recurren para tomar decisiones y conformar su conocimiento práctico.

Es interesante y muy útil para la presente investigación, evidenciar el problema básico de este estudio, puesto que su configuración, desde la exploración del conocimiento práctico del profesorado, en fase de práctica pedagógica de la Escuela Universitaria del Magisterio de la Universidad de la Laguna en Tenerife ${ }^{4}$ se relaciona con lo que se propone esta investigación, en cuanto a la comprensión de las creencias, desde lo que hacen las maestros en su jornada de trabajo.

\footnotetext{
${ }^{4}$ Isla ubicada en el Océano Atlántico, perteneciente a la Comunidad Autónoma de Canarias. Es la más poblada de España y del Archipiélago Canario.
} 
Las conclusiones de dicho trabajo también se constituyen en un aporte valioso puesto que se evidencia la relación directa entre pensamiento y acción y cómo se determinan mutuamente:

- Los futuros profesores construyen representaciones del mundo que les rodea. Dichas representaciones, junto con las experiencias, las interacciones, los conocimientos y demás aspectos que han configurado al sujeto, dan lugar a las teorías implícitas. Estas teorías presentes incluso antes del ingreso a las escuelas de formación docente, inciden de manera directa en su formación y en su quehacer pedagógico.

- Las teorías implícitas sobre la enseñanza del futuro profesorado difieren, tanto cualitativa como cuantitativamente, de aquellas consideradas como teorías formales sobre la enseñanza y los futuros docentes recurren indistintamente a una u otra, dependiendo de la tarea o problema de enseñanza al que se enfrentan, mostrando cierto carácter dinámico y flexible de sus teorías dentro de ejes de racionalidad bien determinados.

El aporte más importante del estudio mencionado a la presente investigación está orientado a la forma en que el profesorado interpreta sus acciones y la realidad, a través de sus propios procesos de pensamiento, los cuales están determinados por la cultura.

Otro estudio de una gran riqueza y que no se puede dejar de citar por la gran contribución en cuanto a narrativas del maestro, se refiere a la investigación de Londoño (2005), El maestro demediado. El maestro sujeto dividido en sus interpretaciones sobre la práctica de la docencia en escuelas primarias y secundarias colombianas, propone la clasificación de las narrativas de los maestros en dos planos diferentes: 1. El ideal, o el que muestra las representaciones que identifica y las metas que se plantea para su futuro y 2. El factual, o los hechos que se dan en la práctica docente y en el desempeño diario de su labor, sumados a las distintas experiencias que el maestro va acumulando durante sus años de docencia.

En los resultados de este estudio se encuentra que los dos planos, el ideal y el factual, no se consideran como planos aislados e independientes uno del otro, sino como dos fuentes de información cultural con la cual el maestro construye su subjetivi- dad. Además devela que el discurso del maestro se moviliza y resignifica a partir de la construcción dialéctica marcada por la experiencia acumulada con los años.

Lo anterior conlleva a una reflexión sobre un aspecto que es esencial para quien desarrolla esta investigación y es el siguiente: cuando se estudian las creencias no se puede dejar de pensar en la importancia del lenguaje, pues éste es un punto álgido en la construcción social de la realidad. Como lo plantean Puerto y González (2005), la narración oral o escrita es un elemento esencial para la recolección de la información, por medio de esta se puede dar cuenta del sujeto, su pensamiento y su realidad.

La narración, al ser una construcción del sujeto a partir de su interacción, pierde objetividad de ser una realidad externa, por lo tanto, más que reflejar la realidad, evidencia el sentido de lo que es verdad para los sujetos, por ello comprender e interpretar los discursos que subyacen a la práctica pedagógica del profesorado, es fundamental para identificar las creencias de los docentes, dentro de una realidad específica, es reconocerlos desde su cultura y su lenguaje, aspecto que le otorga al propio investigador, un panorama en que se resignifica y se llena de sentido su propio quehacer.

En la misma vía, el trabajo desarrollado por Fandiño (2001) de naturaleza exploratoria, busca indagar las concepciones y prácticas en el uso del juguete de cuatro maestras de preescolar, a partir de su discurso y de las acciones que desarrollan dentro de los jardines.

En este estudio las cuatro maestras son de entornos completamente diferentes y es precisamente ello lo que determina también que las creencias sean distintas, pues las realidades desde las cuales se configuran no son las mismas, por ello la presente investigación intenta comprender la creencia desde ese contexto desde el cual emerge,

[...] La forma en que la gente se comporta en una comunidad es a menudo muy diferente de cómo se comporta en otra comunidad [...] pertenecen a diferentes discursos históricos y prácticas sociales que nacen precisamente de diferentes caldos de «cultivo» y, en consecuencia, de diferentes «culturas», para la emergencia de distintos tipos de individuos (Echeverría, 1993, p. 55). 
Una investigación de corte muy similar a la anterior es la desarrollada por Durán (2008), Creencias acerca del juego y el uso de los juguetes en los estudiantes de I semestre de Educación Infantil de la Universidad Pedagógica Nacional (UPN). En esta investigación se pretende, desde el juego y el uso del juguete, identificar y comprender las creencias con las que ingresan los estudiantes de I semestre al espacio académico de Lúdica y Psicomotricidad en la Licenciatura en Educación Infantil de la Universidad Pedagógica Nacional y la movilización que se da en sus creencias, durante el curso, sin pretender decir que es lo único que genera movilización, puesto que la práctica que se desarrolla con niños de 0 a 4 años, puede también ser un factor determinante en este proceso.

Los aportes más significativos de este trabajo para la investigación son los siguientes: en primera instancia el reconocimiento de las creencias acerca del juego y el juguete en los estudiantes de I semestre se hacen explicitas, tanto en lo que hacen como en lo que dicen y las más evidentes al ingreso del espacio son: el juego es un instrumento o herramienta que debe posibilitarle al maestro la enseñanza de conceptos, sobre todo conceptos matemáticos como figuras geométricas, tamaños; también en el juego se deben abordar procesos que tienen que ver con clasificación, seriación, secuencia.

En segunda instancia es el reconocimiento de que las creencias con las que ingresan los estudiantes, permiten iniciar el proceso de movilización, es decir, este es el punto de partida para trabajar lo concerniente a juego y juguete, dentro del espacio académico.

En última instancia una creencia que se moviliza es la del uso del juego y el juguete desde lo instrumental y en consecuencia, casi que exclusivamente para fines muy escolarizados, se comienzan a pensar e incluir en el juego, la exploración, la representación, la interacción y a estructurar juegos en relación con la vida cotidiana de los niños, según los contextos en donde se desarrolla la práctica.

En este trabajo, como en los anteriores se hace evidente que para lograr identificar las creencias y la movilización de las mismas en unos sujetos, es necesario reconocer que el "lenguaje" como producto y herramienta cultural, es un elemento clave en la in- teracción socio-histórica y cultural, puesto que permite crear, recrear y recrearse en una comunidad, construirla, reconstruirla y transformarla.

Finalmente otro estudio a destacar es el desarrollado en Eslovaquia por Zbirková (1999), El juego y el juguete en la herencia cultural de una nación, es una investigación arqueológica histórica, en la cual se tienen en cuenta las concepciones del juego y el juguete en años o siglos anteriores, para poder comparar y analizar sus observaciones. Esta investigación de tipo documental muestra como resultados más contundentes que en el pasado se valoraba el juego organizado, las obras de teatro escolares, el juego didáctico y la pedagogía de la dramaturgia.

Este estudio permite visualizar por una parte que el juego, se ha hecho tradición y cultura en los seres humanos y por otra que la realidad social es reproducida por la gente que actúa desde los propios significados según el contexto.

Todo el panorama anterior presenta una estrecha relación con algunos principios desde los cuales $\mathrm{Pa}$ jares (1992) sintetizó los resultados de la investigación de las creencias de los profesores, y a los cuales se hace referencia a continuación pues muestran un horizonte interesante para esta investigación:

1. Los individuos desarrollan un sistema de creencias que estructura todas las creencias adquiridas a lo largo del proceso de transmisión cultural.

2. Los sistemas de creencias tienen una función adaptativa al ayudar al individuo a definir y comprender el mundo y a sí mismos.

Cuando se afirma que dichos principios configuran un horizonte para la presente investigación, es porque en ellos se menciona el sistema de creencias, que tiene que ver con el hecho de que las creencias no están desarticuladas, sino que por el contrario hay una afectación e incidencia mutua entre ellas, y por eso es necesario comprenderlas como una red, como una estructura, como un sistema: el sistema de creencias se constituye desde los significados que caracterizan las acciones y los discursos del docente, es decir, que la situación en la que se generan las acciones, no es en sí misma lo más interesante, sino cómo el profesor la percibe, actúa y habla de ella; estos significados al organizarse conforman redes que 
a su vez constituyen una estructura, que se consolida a través de un sistema, desde el cual el maestro habla de un campo de conocimiento determinado. Rokeach (1968) al respecto manifiesta que "el sistema de creencias representa el universo total de creencias de una persona acerca del mundo físico, del social y de ella misma".

Desde el punto de vista de la estructura, Llinares (1992) trabaja en torno a tres constructos psicológicos, las ideas núcleo, las razones y las perspectivas de acción que se identifican a través de las siguientes características:

- Idea núcleo: principios, ideas básicas a través de los cuales se apoya y se articula el sistema conceptual del docente.

- Perspectivas de acción: descripción de una acción deseable para la consecución de unos objetivos derivados de las ideas núcleo.

- Razones: declaraciones que pueden apoyar el establecimiento de las ideas núcleo y las perspectivas de acción.

Estos tres constructos son el referente para evidenciar y comprender la complejidad del sistema de creencias sobre juego, el cual se inscribe y se identifica desde sus acciones y sus discursos, pues es de esta manera, como se logra hacer una aproximación al pensamiento del profesor tal como lo muestran los trabajos citados anteriormente, en donde se legitima al docente como un sujeto que al reflexionar su práctica la resignifica.

Para finalizar, es pertinente mostrar las tres premisas que caracterizan el interaccionismo simbólico según Blumer (1982) y la relación que hay entre el estudio de las creencias y el enfoque que alimenta la postura de quien desarrolla esta investigación.

1. El sujeto guía sus actos hacia las cosas, en función de lo que éstas representan para él.

2. El sentido de estas cosas surge como resultado de la interacción social.

3. Los significados se transforman por medio de un proceso interpretativo desarrollado por el sujeto, al enfrentarse con las situaciones con las que se encuentra en el camino.

Dichas premisas muestran la importancia de los significados y su sentido desde un proceso interpretativo, que profesores en ejercicio realizan en una situación determinada. Hay que destacar que la interpretación para el interaccionismo simbólico, es como un proceso formativo en el que los significados son usados y estudiados como elementos que orientan las acciones.

\section{El Juego: aproximación desde la perspectiva socio cultural}

Las prácticas de los maestro, guardan una estrecha relación con enfoques a través de los cuales se piensa el juego en el aula, ya sea como herramienta, como estrategia, cómo fin en sí mismo o como elemento de la cultura que constituye el sujeto; se podría decir que la manera como se ha trabajado y estudiado el juego a partir de diferentes enfoques, alimenta de alguna manera las creencias del profesorado, puesto que algunos, más que otros han tenido una gran incidencia en la educación.

En ese sentido reconocer los enfoques desde los cuales se aborda el juego, permite ubicar y reconocer el lugar del docente en el juego, la concepción del niño y niña que subyace en cada enfoque, comprender la manera como el docente planifica sus acciones y la forma como dispone ambientes para generar juego y los objetos que usa y cómo los organiza, puesto que en cada una de las perspectivas, se construyen unas determinadas interacciones, unos itinerarios y discursos particulares.

Para comenzar este recorrido, es importante hacer alusión al juego desde algunos trabajos que se han desarrollado desde la una perspectiva sociocultural, puesto que a partir de allí el juego es conceptualizado como una práctica social que informa sobre la organización ideológica, cultural y mental de las sociedades, pensar el juego de esta manera ha comenzado a abrir un nuevo camino en el escenario educativo, para reconocerlo y comprenderlo, más allá de lo instrumental, para pensar que las variaciones culturales, llevan a cambios en los modos de pensar y representarse la realidad, y que estos cambios son mediados por el lenguaje, por las categorías perceptuales, por distintos sistemas de valores.

En ese sentido a continuación se referencian algunos autores que se han dedicado a pensar el juego como una dimensión que constituye al ser humano y como práctica social y cultural, para comprenderlo más que como un medio, como un fin en sí mismo. 
En primer lugar se presenta a Martha Glanzer (2000) quien expresa que los juegos han sido infinitamente variados y que las diferentes comunidades los han ido marcando con sus características étnicas y sociales específicas. Ella hace un planteamiento muy interesante, puesto que manifiesta que el niño refleja en sus conductas y en sus juegos las particularidades que caracterizan una determinada sociedad contextualizada en un determinado momento histórico. Esta autora hace pensar que los juegos se relacionan por medio de las normas y significados con el sistema de creencias de una sociedad, en otras palabras que el juego como un lenguaje, tiene una gran riqueza semántica, que al ser analizada muestra las especificidades de una cultura.

En ese sentido Huizinga (1987) historiador holandés del siglo XX plantea que la cultura humana emerge del juego: "no se trata [...] del lugar que al juego corresponda entre las demás manifestaciones de la cultura, sino en qué grado la cultura misma ofrece un carácter de juego". Su intencionalidad lleva a este importante autor ampliar la noción de juego más allá de la niñez, hasta el conjunto de las manifestaciones humanas, y ve en él las relaciones con la máscara, los mitos. Igualmente, Huizinga establece una relación profunda con la estética, el arte barroco, la "fantasía" romántica...

El juego, en su aspecto formal, es una acción libre ejecutada "como si" y sentida como situada fuera de la vida corriente, pero que, a pesar de todo, puede absorber por completo al jugador, sin que haya en ello ningún interés material ni se obtenga en ella provecho alguno, que se ejecuta dentro de un determinado tiempo y un determinado espacio, que se desarrolla en un orden sometido a reglas y que da origen a asociaciones que propenden a rodearse de misterio o disfrazarse para destacarse del mundo habitual (Huizinga,1987).

El estudio de Callois (1986) amplía lo esbozado por Huizinga, en lo que se refiere a las características del juego y de manera muy explícita, aclara que este tiene unas condiciones, unas particularidades, una estructura flexible y abierta, pero que da un marco para pensar que no se puede catalogar como juego a todas las actividades que en el caso de las instituciones, realiza el niño en el aula, porque además afirma: el jugador no puede estar obligado sin que el juego pierda al punto su naturaleza de diversión atractiva y alegre; el desarrollo del juego no puede estar pre- determinado, ni el resultado dado de antemano, por dejarse obligatoriamente a la iniciativa del jugador cierta libertad en la necesidad de inventar; por último se refiere al carácter de improductividad del juego, reconociendo que en él, no se crean en él, ni bienes, ni riqueza, ni tampoco elemento nuevo de ninguna especie.

Las características del juego precisadas por Huizinga y Callois le han dado una mirada diferente al juego en la educación, y esta ha sido tan seductora, que ha comenzado a reflejarse en los discursos que circulan en las instituciones sobre todo de Educación Inicial, no obstante especialmente el asunto de la libertad en el juego, es muy ambiguo; puesto que muchas veces se tiende a confundir la libertad con el abandono del juego, quizás el maestro al querer respetar su espontaneidad, no lo acompaña por temor arruinar el mismo juego y porque además no hay certeza de cómo se acompaña el juego libre en muchos de esos discursos.

Continuando con la perspectiva socio-cultural, está la investigación desarrollada por Del Castillo (2001) Juegos, diálogos, historia puesto que conduce a encontrar los puntos de unión entre los tres aspectos mencionados, pues por una parte evidencian el diálogo como juego y la historia como diálogo y por otra analizan el concepto de juego, como construcción social, histórica y cultural.

Un gran complemento para lo anterior es la investigación Para una sociología del juego, de López (1994), en la que se desarrolla un estudio sobre las pautas estructurales y funcionales de conducta, en el campo del juego, estableciendo, toda una gama de variables que explican las causas y consecuencias que conlleva la existencia, mantenimiento, y futuro del juego hoy, como factor de cambio social. En este trabajo, se considera que el juego cumple unas funciones específicas en la vida del individuo, como factor de aprendizaje y socialización. En el juego entonces, el niño accede a la vida de relación con los demás y a la cultura, sin arriesgar su infancia conservando ese bagaje subjetivo, personal, que diferencia a cada ser humano al situarse en la frontera de lo social.

En ese sentido en la investigación de Prieto (1995), El juego simbólico: su valor como agente de socialización en la vida del niño, se plantea como hipótesis centrales las siguientes: en primera instancia que el juego simbólico permite al niño trascender 
a la realidad exterior de lo social y al mundo de la cultura; pasar de la sensación al pensamiento; de la representación intelectual a los esquemas de la vida social; y en segunda instancia que el juego simbólico permite verificar en el niño y la niña, el tránsito de la subjetividad a la objetividad de la vida social se concluye, que ese juego, entre los 2-7 años, se inserta en la vida del niño como un intermedio entre la personalización y la socialización, proporcionando libertad e iniciativa personal en un contexto social y cultural determinado.

Es de gran valor en este estudio el análisis fenomenológico que se desarrolla, puesto que en dicho análisis cobra importancia el conocimiento de sentido común de los sujetos sociales, en tanto en él se incorporan las interpretaciones que los sujetos realizan, para dar sentido a los fenómenos y otorgar significados a sus acciones reconociendo que la comprensión del mundo que hacen los sujetos es el resultado de intercambios entre la gente, históricamente situada.

La forma en que nos damos un sentido y la forma en que actuamos en la vida no es arbitraria... Las formas como conferimos sentidos y como actuamos descansan tanto en la historia como en las prácticas vigentes de la comunidad a que pertenecemos (Echeverría,1993, p. 54).

El fragmento anterior conduce a valorar esos legados históricos y culturales que constituyen al sujeto y sus creencias, en ese sentido Bantulà (2006) en la investigación documental Los estudios socioculturales sobre el juego tradicional: una revisión taxonómica, examina los aportes de la antropología al estudio del juego como ámbito específico dentro de la disciplina, el autor hace hincapié en las diversas taxonomías que se han formulado para sistematizar el conjunto de manifestaciones lúdicas.

En el estudio mencionado se destaca el proceso que se gesta entre el investigador, lo observado y la sociedad, articulándose en forma totalizadora, interactiva y versátil, con el único propósito de conocer y comprender la realidad del juego y conceptualizarla de una manera sistémica, holística, global y dinámica.

Por último y desde una perspectiva de género y en la cual se concibe al sujeto desde la integración dialéctica en una realidad económica, social, polí- tica y cultural determinada por la "interacción con otros", se encuentra la tesis de Lobato (2007) quien desarrolla la investigación Juego y género, cuyo objetivo primordial es ampliar el conocimiento sobre la influencia del juego infantil en la construcción de la identidad de género en los contextos y prácticas infantiles.

\section{Referencias}

Argoz, J. (1997). El pensamiento del profesor de educación infantil: Estudio de casos sobre el conocimiento práctico de docentes en ejercicio, Oviedo, Tesis Doctoral, Universidad de Oviedo.

Bantulà, J. (2006). 'Los estudios socioculturales sobre el juego tradicional: una revisión taxonómica'. En Revista de Dialectología y Tradiciones Populares 2, 19-42.

Blumer, H. (1982). El interaccionismo simbólico: perspectiva y método, Barcelona, Hora S.A.

Callois, R. (1986). Los juegos y los Hombres, México, Fondo de Cultura Económica.

Clark, M. C. y Peterson, P. (1986-1990). 'Procesos de pensamiento de los docentes'. En Wittrock, M. La investigación de la enseñanza III, Barcelona, Cap. 6, Paidós.

Del Castillo, R. (2001). 'Juegos, diálogos, historia'. En Revista Volubilis 9, 77-95.

Dewey, J. (1933 - 1989). Cómo pensamos. Nueva exposición de la relación entre pensamiento reflexivo y proceso educativo, Barcelona, Paidós.

Durán, S. (2008). Creencias acerca del juego y el juguete en estudiantes de I semestre de la Licenciatura en Educación Infantil de la Universidad Pedagógica Nacional, Tesis de maestría, Facultad de Educación, Universidad Pedagógica Nacional.

Echeverría, B. (1993). Formación profesional. Guía para el seguimiento de su evolución, Barcelona, Promociones y Publicaciones Universitarias.

Fandiño, G. (2001). 'El uso del juguete en los jardines infantiles'. En Revista Pedagogía y Saberes 16, 71-79.

Fandiño, G. (2004). El pensamiento del profesor sobre la planificación dentro del trabajo por proyectos en el grado de transición: estudio de caso, Madrid, Tesis Doctoral, Universidad Nacional de Educación a distancia, UNED.

Fenstermacher, G.R. (1989). 'Tres aspectos de la filosofía de la investigación sobre la enseñanza'. En Wittrock, M.C. (Comp.). En La investigación de la enseñanza I. Enfoques, teorías y métodos, Barcelona, Paidós.

Gallego, G. (1991). 'Investigación sobre pensamiento del profesor: aproximaciones al estudio de las teorías y creencias de los profesores'.En Revista Española de pedagogía XLIX, 189.

Glanzer, M. (2000). El juego en la niñez, Buenos Aires, Aique. 
Huizinga, A. (1954/1987). Homoludens, Madrid, Emecé, segunda reimpresión.

Laberee, Smyth, Zechner y otros (1999). Desarrollo profesional del docente: política, investigación y práctica, Reino Unido, Ediciones Akal. Capítulo El análisis de las creencias del profesorado como requisito de desarrollo profesional. Miguel Sola Fernández. (XXII) 661-683.

Llinares, S. (1992). 'Los mapas cognitivos como instrumento para investigar las creencias epistemológicas de los profesores'. En Marcelo, C. La investigación sobre formación del profesorado: métodos de investigación y análisis de datos, Argentina, Cincel, 49-57.

Lobato, E. (2007). Juego y genero. Estudio etnográfico sobre la construcción de la identidad de género en los contextos y prácticas lúdicas infantiles, Tesis Doctoral, Facultad de Educación, Universidad de Oviedo.

Londoño, S. (2005). El maestro demediado. El maestro sujeto dividido en sus interpretaciones sobre la práctica de la docencia en escuelas primarias y secundarias colombianas, Tesis de Maestría, Facultad de Antropología, Universidad de los Andes.

López, M. (1994). Juegos y educación en el tiempo libre infantil, Tesis Doctoral, Facultad de Educación, Universidad Pontificia Comillas.

Marcelo, C. (1992). La investigación sobre formación del profesorado: métodos de investigación y análisis de datos, Sevilla, Cincel.
Martínez, J. (1994). Las teorías implícitas y la resolución de problemas de la enseñanza: el pensamiento del profesorado en formación inicial, Tesis Doctoral, Facultad de Educación, Universidad de La Laguna.

Pajares, F. (1992). 'Teachers' beliefs and educacional research: cleaning up messy construct'. En Review of educational Research, Vol. 62, No 3, 307-332.

Prieto, A. (1995). El juego simbólico: su valor como agente de socialización en la vida del niño, Tesis Doctoral, Facultad de Educación, Universidad Nacional de Educación a Distancia.

Puerto, G. y González, I. (2005). 'Mujeres marroquíes en Castilla-La Mancha: algunas conclusiones de una investigación'. En Izquierdo Brichs, Ferran y Desrues, Thierry (coords.) (2005). Actas del Primer congreso del Foro de Investigadores sobre el Mundo Árabe y Musulmán (FIMAM), Barcelona, Fimam.

Zbirková, V. (1999). 'El juego y el juguete en la herencia cultural de una nación'. En Correo del Maestro 43, Naucalpan: Uribe y Ferrari Editores S. A. Obtenido el 10 de Marzo de 2008, desde: www.correodelmaestro.com/ anteriores/1999/diciembre43/3anteaula43.htm 